\title{
GENERAL RELATIVITY DERIVATION OF BEAM REST-FRAME HAMILTONIAN*
}

\author{
J. Wei ${ }^{\dagger}$, Brookhaven National Laboratory, New York, USA
}

\begin{abstract}
Analysis of particle interaction in the laboratory frame of storage rings is often complicated by the fact that particle motion is relativistic, and that reference particle trajectory is curved. Rest frame of the reference particle is a convenient coordinate system to work with, within which particle motion is non-relativistic. We have derived the equations of motion in the beam rest frame from the general relativity formalism, and have successfully applied them to the analysis of crystalline beams [1].
\end{abstract}

\section{DERIVATION}

The motion of charged particles under Coulomb interaction and external electromagnetic (EM) forces can be most conveniently described in the rotating rest frame of the reference particle of which the orientation of the axes are constantly aligned to the radial, tangential, and vertical direction of the motion. In this frame, particle motion within the beam bunch is non-relativistic.

We derive the equations of motion using the general relativity formalism. First, we express the equations of motion in a general tensor formalism. The Lorentz force experienced by the particle is constructed as a product of the EM field tensor and the four-velocity. Starting from the laboratory frame, the EM field tensor is written by means of the components of the EM fields. Then, tensor algebra is used to transform this field tensor into the rest frame. With a similar transformation, the metric tensor of the rest frame is also obtained. The equations of motion can thus be constructed in the rest frame which include centrifugal force, Coriolis force, time-dependent external EM forces, and electrostatic Coulomb forces. Finally, these equations are re-scaled in terms of dimensionless quantities for the convenience of computer simulation and analysis.

\subsection{Tensor formalism}

Adopting the formalism used by Møller,[2] we consider the motion of a particle in an arbitrary system of coordinates $\left(x^{i}\right)$, where $i=1,2,3,4$ indicate the space-time components. The metric tensor $\left\{g_{i j}\right\}$ is defined in terms of the differential line element $d s$ as $d s^{2}=g_{i j} d x^{i} d x^{j}$, where the summation is performed over the four indices. Let $x^{i}=x^{i}(\tau)$ be the equation of the time track of the motion, $\tau$ being the proper time of the particle. The contravariant components of the four-velocity are

$$
U^{i} \equiv \frac{d x^{i}}{d \tau}=\left(\Gamma u^{\iota}, \Gamma c\right)
$$

\footnotetext{
* Work performed under the auspices of the US Department of Energy
}

$\dagger$ wei1@bnl.gov, on joint appointment at ORNL and BNL where $u^{\iota}=d x^{\iota} / d t$ are the contravariant components of the spatial velocity, the Greek letter $\iota$ indicates the three spatial components, $d t=\Gamma d \tau, c$ is the speed of light, and

$$
\Gamma=\left\{\left[\left(-g_{44}\right)^{\frac{1}{2}}-\frac{g_{\kappa 4} u^{\kappa}}{c \sqrt{-g_{44}}}\right]^{2}-\frac{u^{2}}{c^{2}}\right\}^{-\frac{1}{2}}
$$

is the generalized Lorentz factor. The contravariant components of the four-momentum are $P^{i}=m_{0} U^{i}$, where $m_{0}$ is the proper mass of the particle.

Acted upon by a non-gravitational four-force $\left\{F_{i}\right\}$, the equations of motion of the particle can be written as

$$
\frac{D P^{i}}{d \tau}=g^{i k} F_{k}, \quad F_{i} U^{i} \equiv 0,
$$

where the $4 \times 4$ matrix $\left\{g^{i j}\right\}$ is the inverse of $\left\{g_{i j}\right\}$, and the covariant differentiation is defined as

$\frac{D P^{i}}{d \tau} \equiv \frac{d P^{i}}{d \tau}+\Gamma_{i k}^{l} U^{k} P^{l}, \Gamma_{i k}^{l}=\frac{g^{l m}}{2}\left(\frac{\partial g_{m i}}{\partial x^{k}}+\frac{\partial g_{m k}}{\partial x^{i}}-\frac{\partial g_{i k}}{\partial x^{m}}\right)$

Among the non-gravitational forces, the external EM force acting upon the particles is expressed by means of the EM field tensor $\left\{F_{i j}\right\}$ as

$$
F_{i}=\frac{e}{c} F_{i k} U^{k},
$$

where $e$ is the electric charge, and $\left\{F_{i j}\right\}$ is anti-symmetric. The Maxwell's equations are given by

$$
\left\{\begin{array}{l}
\frac{\partial F_{i k}}{\partial x^{l}}+\frac{\partial F_{k l}}{\partial x^{i}}+\frac{\partial F_{l i}}{\partial x^{k}}=0 \\
\frac{1}{\sqrt{|g|}} \frac{\partial}{\partial x^{i}}\left(\sqrt{|g|} F^{i k}\right)=\frac{\rho_{0} U^{i}}{c},
\end{array}\right.
$$

where $F^{i j}=g^{i l} g^{j m} F_{l m},|g|$ is the absolute value of the determinant of the metric $\left\{g_{i j}\right\}$, and $\rho_{0}$ is the charge density measured in the system of inertia.

\subsection{Laboratory frame}

In the Cartesian coordinate system of inertia $\left(X^{i}\right)$, the so-called laboratory frame, Eq. 2 can be written in the conventional vector form. In this frame, the only non-zero components of the metric tensor are

$$
g_{11}=g_{22}=g_{33}=1, \text { and } g_{44}=-1 .
$$

In terms of the conventionally defined electric and magnetic fields

$$
\mathbf{E}=\left(E_{1}, E_{2}, E_{3}\right), \quad \mathbf{B}=\left(B_{1}, B_{2}, B_{3}\right),
$$


the EM field tensor is

$$
\left\{F_{i j}\right\}=\left(\begin{array}{rrrr}
0 & B_{3} & -B_{2} & E_{1} \\
-B_{3} & 0 & B_{1} & E_{2} \\
B_{2} & -B_{1} & 0 & E_{3} \\
-E_{1} & -E_{2} & -E_{3} & 0
\end{array}\right) .
$$

It is straightforward with these expressions to verify that Eq. 2 is equivalent to the equations

$$
m_{0} \frac{d(\Gamma \mathbf{u})}{d t}=e \mathbf{E}+\frac{e}{c} \mathbf{u} \times \mathbf{B}, m_{0} c^{2} \frac{d \Gamma}{d t}=e \mathbf{u} \cdot \mathbf{E},
$$

where $d t=\Gamma d \tau$, and $\Gamma=\left(1-u^{2} / c^{2}\right)^{-1 / 2}$ is in this case the Lorentz factor.

\subsection{Beam rest frame of fixed orientation}

Consider a reference particle circulating around the vertical axis $X^{2}$ with a uniform angular velocity $\omega$ at a radius $R$. Its time track $\left\{f^{i}\right\}$ may be described in the laboratory frame in terms of the proper time $\tau$

$$
f^{i}(\tau)=(R \cos \theta, 0, R \sin \theta, \gamma \tau),
$$

where $\theta=\omega \gamma \tau$ is the revolution angle, $\gamma=\left(1-\beta^{2}\right)^{-1 / 2}$, and $\beta c=R \omega$ is the velocity. Introduce a rigid system of coordinates $\left(x^{i}\right)$ which follows the reference particle in its motion, so that the particle is constantly situated at the origin of this frame of reference, and that the spatial axes have constant orientations. The transformation connecting the variables $\left(X^{i}\right)$ and $\left(x^{i}\right)$ are

$$
X^{i}=f^{i}(\tau)+\check{\alpha}_{i \kappa} x^{\kappa}, \text { and } x^{4}=\tau,
$$

where the check ( $)$ denotes the inverse operation, the summation over $\kappa$ is on spatial components $1,2,3$. The coefficients $\alpha_{i j}$ are obtained by means of successive infinitesimal Lorentz transformations without rotation of the spatial axes,[2]

$$
\left\{\alpha_{i j}\right\}=\left(\begin{array}{cccc}
\alpha_{11} & 0 & \alpha_{13} & \gamma \beta \sin \tilde{\theta} \\
0 & 1 & 0 & 0 \\
\alpha_{31} & 0 & \alpha_{33} & -\gamma \beta \cos \tilde{\theta} \\
\gamma \beta \sin \theta & 0 & -\gamma \beta \cos \theta & \gamma
\end{array}\right),
$$

where $\alpha_{11}=\cos \theta \cos \tilde{\theta}+\gamma \sin \theta \sin \tilde{\theta}, \alpha_{13}=\sin \theta \cos \tilde{\theta}-$ $\gamma \cos \theta \sin \tilde{\theta}, \alpha_{31}=\cos \theta \sin \tilde{\theta}-\gamma \sin \theta \cos \tilde{\theta}, \alpha_{33}=$ $\sin \theta \sin \tilde{\theta}+\gamma \cos \theta \cos \tilde{\theta}$, and $\tilde{\theta}=\omega \gamma^{2} \tau=\gamma \theta$. The fact that $\tilde{\theta}$ differs from the revolution angle $\theta$ by the Lorentz factor $\gamma$ is the consequence of the Thomas precession.

To obtain the metric tensor of the rest frame, we need to derive the relation between the line elements of the two coordinate systems. Differentiation of Eq. 11 gives

$$
d X^{i}=\check{\alpha}_{i \kappa} d x^{\kappa}+\left[\frac{f^{i}(\tau)}{d \tau}+\frac{\check{\alpha}_{i \kappa}(\tau)}{d \tau} x^{\kappa}\right] d \tau \equiv \check{\alpha}_{j}^{i} d x^{j},
$$

where $\left\{\alpha_{j}^{i}\right\}=\left\{\check{\alpha}_{j}^{i}\right\}^{-1}$ is the transformation matrix between the line elements of the laboratory and the rest frame. Let the prime (') denote the rest frame. The metric tensor $\left\{g_{i j}^{\prime}\right\}$ of the rest frame is related to $\left\{g_{i j}\right\}$ of the laboratory frame by the relation $g_{i j}^{\prime}=\check{\alpha}_{i}^{l} \check{\alpha}_{j}^{m} g_{l m}$. Using the transformation matrix, the contravariant components of a vector $\left\{a^{i}\right\}$ in the laboratory frame are transformed into the rest frame as $a^{\prime} i=\alpha_{k}^{i} a^{k}$. On the other hand, the covariant components of a tensor of rank 2, e.g. the EM field tensor, are transformed as $F_{i j}^{\prime}=\check{\alpha}_{i}^{l} \check{\alpha}_{j}^{m} F_{l m}$. With these relations, the equation of motion in the beam rest frame of fixed orientation can be obtained from Eq. 2 by an explicit evaluation.

\subsection{Rotating beam rest frame}

The equations of motion can be greatly simplified in terms of the variables tangential and normal to the direction of the motion of the reference particle. We thus seek for another transformation into the rotating beam rest frame of which the orientation of the spatial axes are constantly aligned to the radial $(x)$, tangential $(z)$, and vertical $(y)$ direction of the motion of the reference particle.

Define the rotating beam frame as the rest frame of which the orientations of the spatial axes $x$ and $z$ rotate relative to those of the fixed orientation $x^{1}$ and $x^{3}$

$$
\left(\begin{array}{l}
x \\
y \\
z \\
\tau
\end{array}\right)=\left(\begin{array}{cccc}
\cos \tilde{\theta} & 0 & \sin \tilde{\theta} & 0 \\
0 & 1 & 0 & 0 \\
-\sin \tilde{\theta} & 0 & \cos \tilde{\theta} & 0 \\
0 & 0 & 0 & 1
\end{array}\right)\left(\begin{array}{l}
x^{1} \\
x^{2} \\
x^{3} \\
x^{4}
\end{array}\right) .
$$

Express the electric and magnetic fields in the laboratory frame in terms of the tangential component in $z$ and the normal component in $x$

$$
\begin{array}{ll}
E_{x}=E_{1} \cos \theta+E_{3} \sin \theta, & B_{x}=B_{1} \cos \theta+B_{3} \sin \theta \\
E_{y}=E_{2}, & B_{y}=B_{2} \\
E_{z}=-E_{1} \sin \theta+E_{3} \cos \theta, & B_{z}=-B_{1} \sin \theta+B_{3} \cos \theta
\end{array}
$$

The equations of motion can be obtained from Eqs. 2 and 3 by using Eq. 8 and the transformation relations as

$$
\left\{\begin{array}{l}
\frac{m_{0}}{\Gamma}\left(\ddot{x}-2 \gamma^{2} \omega \dot{z}-\gamma^{4} \omega^{2} x\right)-m_{0} \Gamma \gamma^{2} \omega^{2} R(1-\chi)= \\
=e(1-\chi) E_{x}^{\prime}+\frac{e}{\Gamma c}\left[B_{z}^{\prime} \dot{y}-B_{y}^{\prime}\left(\dot{z}+\gamma^{2} \omega x\right)\right]-\frac{\partial V}{\partial x}, \\
\frac{m_{0}}{\Gamma} \ddot{y}=e(1-\chi) E_{y}^{\prime}+ \\
\quad+\frac{e}{\Gamma c}\left[B_{x}^{\prime}\left(\dot{z}+\gamma^{2} \omega x-B_{z}^{\prime}\left(\dot{x}-\gamma^{2} \omega z\right)\right]-\frac{\partial V}{\partial y},\right. \\
\frac{m_{0}}{\Gamma}\left(\ddot{z}+2 \gamma^{2} \omega \dot{x}-\gamma^{4} \omega^{2} z\right)= \\
=e(1-\chi) E_{z}^{\prime}+\frac{e}{\Gamma c}\left[B_{y}^{\prime}\left(\dot{x}-\gamma^{2} \omega z\right)-B_{x}^{\prime} \dot{y}\right]-\frac{\partial V}{\partial z},
\end{array}\right.
$$

where

$$
\begin{array}{ll}
E_{x}^{\prime}=\gamma\left(E_{x}-\beta B_{y}\right), & B_{x}^{\prime}=\gamma\left(B_{x}+\beta E_{y}\right) \\
E_{y}^{\prime}=\gamma\left(E_{y}+\beta B_{x}\right), & B_{y}^{\prime}=\gamma\left(B_{y}-\beta E_{x}\right) \\
E_{z}^{\prime}=E_{z}, & B_{z}^{\prime}=B_{z}
\end{array}
$$

are the electric and magnetic fields after a Lorentz transformation without rotation, and $V$ is the electric potential. For particles of the same bunch, the potential $V_{C}$ describing the 
Coulomb interaction is

$$
V_{C}(x, y, z)=\sum_{j} \frac{e^{2}}{\sqrt{\left(x_{j}-x\right)^{2}+\left(y_{j}-y\right)^{2}+\left(z_{j}-z\right)^{2}}}
$$

where the summation is performed over all the other particles $j$ and their image charges. The potential $V_{r f}$ describing a radio-frequency (rf) electric field $E_{s}$ in the laboratory frame satisfies

$$
\frac{\partial V_{r f}(x, y, z)}{\partial z}=-e E_{s}
$$

Since the particle motion in the rest frame is nonrelativistic, the Lorentz factor $\Gamma$ is simplified as

$$
\Gamma \approx(1-\chi)^{-1}, \text { with } \chi \equiv \beta^{2} \gamma^{2} \frac{x}{R} .
$$

Typically, the dimensionless quantity $\chi$ is much smaller than 1. The terms on the left hand side of Eq. 16 include the centrifugal and Coriolis forces. Those on the right hand side are external EM forces and electrostatic Coulomb forces. Again, since the particle motion is non-relativistic, the magnetic force produced by the motion of the particles is negligible compared with the electrostatic force.

\section{STORAGE RING EXAMPLE}

We consider the case that the beam is guided by a bending field $B_{0}$ satisfying

$$
e B_{0} R=m_{0} c^{2} \beta \gamma,
$$

and focused by a quadrupole field of gradient $B_{1}$

$$
B_{x}=B_{1} y, B_{y}=B_{0}+B_{1} x, B_{z}=0,
$$

where $B_{1}$ may vary for different piece of magnets, and the field variation at the magnet end is neglected. The equations of motion is simplified by linearizing Eq. 16

$$
\left\{\begin{array}{l}
m_{0}\left(\ddot{x}-\gamma^{2} \omega \dot{z}\right)-m_{0} \gamma^{2} \omega^{2} R \chi=-e \beta \gamma B_{1} x-\frac{\partial V}{\partial x}, \\
m_{0} \ddot{y}=e \beta \gamma B_{1} y-\frac{\partial V}{\partial y} \\
m_{0}\left(\ddot{z}+\gamma^{2} \omega \dot{x}\right)=-\frac{\partial V}{\partial z} .
\end{array}\right.
$$

Here, $\chi$ has been assumed small compared with 1 .

\subsection{Dimensionless variables}

Eq. 23 can be simplified in form when it is expressed in terms of dimensionless variables. Let $n \equiv-B_{1} R / B_{0}$ represent the strength of the focusing magnetic field, and $\xi \equiv\left(r_{0} R^{2} / \beta^{2} \gamma^{2}\right)^{1 / 3}$ be a characterization of the interparticle distance in the presence of Coulomb interaction in the storage ring, where $r_{0}=e^{2} / m_{0} c^{2}$ is the classical radius of the particle. Express the time $t$ in unit of $R / \beta \gamma c$, the spatial coordinates $x, y$, and $z$ in unit of $\xi$, and the energy in unit of $\beta^{2} \gamma^{2} e^{2} / \xi$. Eq. 23 becomes in these units

$$
\left\{\begin{array}{l}
\ddot{x}-\gamma \dot{z}+\left(-\gamma^{2}+1-n\right) x=-\frac{\partial V}{\partial x} \\
\ddot{y}+n y=-\frac{\partial V}{\partial y} \\
\ddot{z}+\gamma \dot{x}=-\frac{\partial V}{\partial z}
\end{array}\right.
$$

Here, the dots denote differentiations with respect to the normalized time $t$. The normalized Coulomb potential and rf potential satisfy

$$
V_{C}(x, y, z)=\sum_{j} \frac{1}{\sqrt{\left(x_{j}-x\right)^{2}+\left(y_{j}-y\right)^{2}+\left(z_{j}-z\right)^{2}}}
$$

and

$$
\frac{\partial V_{r f}(x, y, z)}{\partial z}=-\frac{e E_{s} \xi}{m_{0} c^{2}}\left(\frac{R}{\xi \beta \gamma}\right)^{2}
$$

In the normalized units, the revolution period of the reference particle in the storage ring is $2 \pi$.

\subsection{Hamiltonians}

Using the canonical momentum $\left(P_{x}, P_{y}, P_{z}\right)$, the particle system of Eq. 24 can be described by the Hamiltonian

$H=\frac{1}{2}\left(P_{x}^{2}+P_{y}^{2}+P_{z}^{2}\right)-\gamma x P_{z}+\frac{1}{2}\left[(1-n) x^{2}+n y^{2}\right]+V$

where the cross term $-\gamma x P_{z}$ describes the coupling between the tangential and normal motion.

\subsection{General case}

The formalism presented in the previous sections can be easily generalized to a storage ring that consists of both bending and straight sections. Denote the guiding field and bending radius in the bending section as $B_{0}$ and $R$, respectively. The equations of motion in the bending sections are given by Eq. 24, while the system Hamiltonian is given by Eq. 27. In the straight sections where the guiding field is zero, the strength $n$ of the focusing field can be defined by normalizing the gradient $B_{1}$ in the straight section to $B_{0}$ in the bending section. The Hamiltonian becomes

$$
H=\frac{1}{2}\left(P_{x}^{2}+P_{y}^{2}+P_{z}^{2}\right)+\frac{1}{2}\left[-n x^{2}+n y^{2}\right]+V
$$

If the circumference of the ring is $C$, the revolution period of the reference particle is $C / R$ in the normalized unit.

\section{DISCUSSIONS}

The equations of motion derived for the beam rest frame made possible direct utilization in storage ring analysis of techniques like the molecular dynamics methods developed typically for non-relativistic systems. These equations can be easily generalized for storage rings containing multipole magnets and multi-species of ions. [3]

Derivations presented in this paper are by-products of a study on crystalline beams in collaboration with A.M. Sessler and X-P. Li.

\section{REFERENCES}

[1] J. Wei, X-P. Li, A.M. Sessler, Phys. Rev. Lett., 73, (1994) 3089.

[2] C. Mфller, The Theory of Relativity, Oxford, 1952.

[3] J. Wei, H. Okamoto, A.M. Sessler, Phys. Rev. Lett., 80, (1998) 2606. 